\title{
Proyectos Escolares Saludables. Descansos Activos. Guía para profesores
}

Documento elaborado por:

Mairena Sánchez López

David Gutiérrez Díaz del Campo

Abel Ruiz de la Hermosa Fernández Infante

Carlos López Vera

Mª Isabel Sánchez Brotons

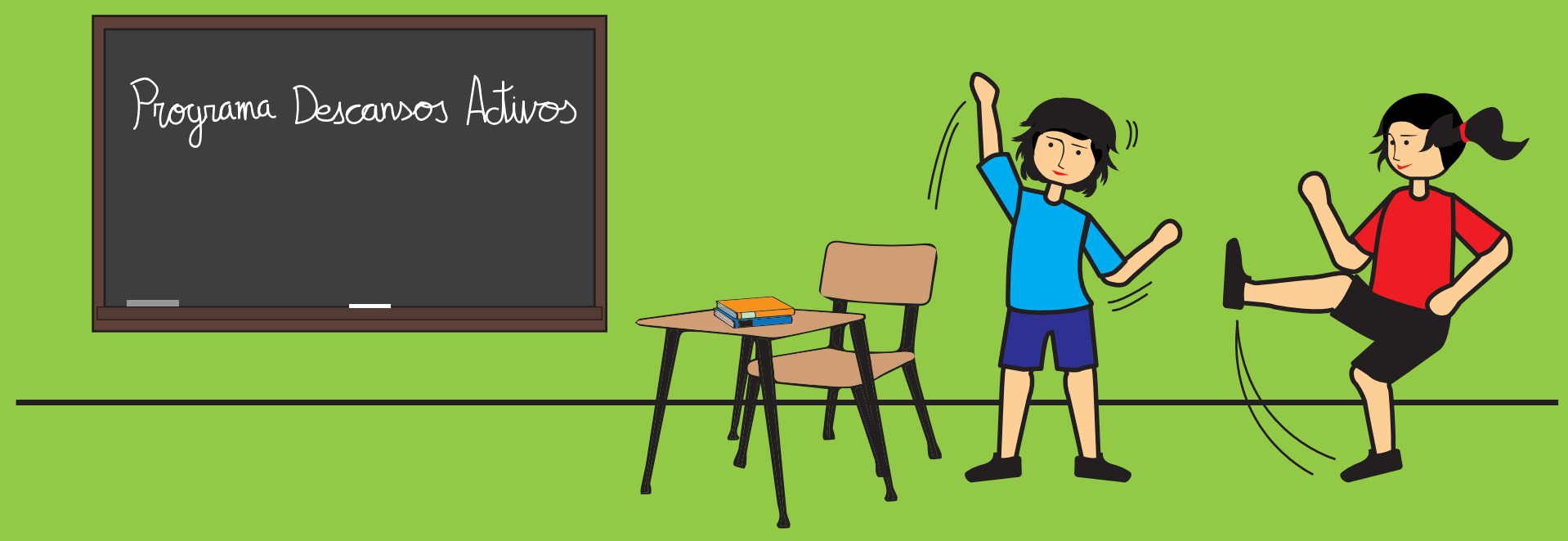

(ิ) $\frac{\text { Ediciones de la Universidad }}{\text { de Castilla-La Mancha }}$ 


\title{
Proyectos Escolares Saludables. Descansos Activos. Guía para profesores
}

\author{
Documento elaborado por: \\ Mairena Sánchez López \\ David Gutiérrez Díaz del Campo \\ Abel Ruiz de la Hermosa Fernández Infante \\ Carlos López Vera \\ $\mathrm{M}^{\mathrm{a}}$ Isabel Sánchez Brotons
}

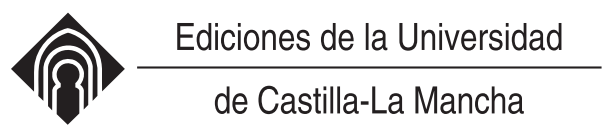

Entidades colaboradoras:

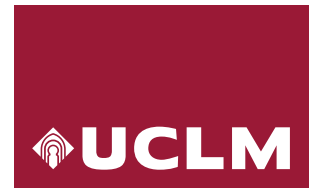

Universidad de

Castilla La Mancha

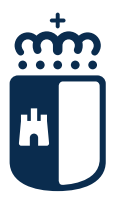

Castilla-La Mancha
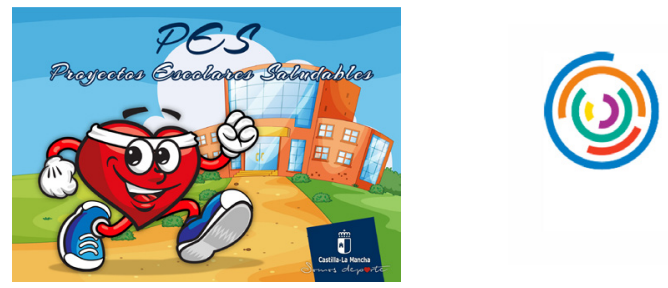
(C) de los textos: sus autores

(C) de la edición: Universidad de Castilla-La Mancha

Edita: Servicio de Publicaciones de la Universidad de Castilla-La Mancha

Colección: ATENEA n ${ }^{\circ} 4$

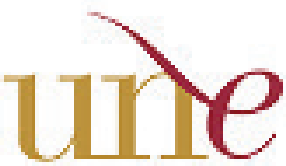

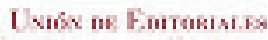

Uxเขenstranias Eispakiols
Esta editorial es miembro de la UNE, lo que garantiza la difusión y comercialización de sus publicaciones a nivel nacional e internacional.

Edición electrónica: http://dx.doi.org/10.18239/atenea.04.2017

Composición: El Perchero

Esta obra se encuentra bajo una Licencia Creative Commons BY-NC-SA 3.0. Cualquier forma de reproducción, distribución, comunicación pública o transformación de esta obra no incluida en la licencia Creative Commons BY-NC-SA 3.0. solo puede ser realizada con la autorización expresa de los titulares, salvo excepción prevista por la ley. Puede acceder Vd. al texto completo de la licencia hacienco click en este enlace: http://creativecommons.org/ licenses/by-nc-sa/3.0/es/legalcode.es 


\section{Documento elaborado por:}

\section{Mairena Sánchez López}

Profesora de la Facultad de Educación de Ciudad Real, Universidad de Castilla-La Mancha.

Investigadora del Centro de Estudios Sociosanitarios de Cuenca, Universidad de Castilla-La Mancha.

\section{David Gutiérrez Díaz del Campo}

Profesor de la Facultad de Educación de Ciudad Real, Universidad de Castilla-La Mancha.

\section{Abel Ruiz de la Hermosa Fernández Infante*}

Graduado en Educación Primaria e Infantil.

Investigador Predoctoral, Universidad de Castilla-La Mancha.

\section{Carlos López Vera*}

Graduado en Educación Infantil.

\section{$\mathbf{M}^{\mathrm{a}}$ Isabel Sánchez Brotons*}

Estudiante del Grado en Educación Infantil.

* Becarios adscritos al proyecto "Descansos Activos para mejorar la salud y el rendimiento académico en los escolares. Adaptación de los materiales del programa TAKE10-CHILE!" (Convenio de colaboración entre la Consejería de Educación, Cultura y Deportes de la JCCM y la UCLM para el desarroIlo de programas de fomento de la actividad física y el deporte). 
En la redacción de los siguientes materiales se ha utilizado el masculino genérico para referirse a los individuos de ambos sexos, sin intención de que esta práctica (avalada por la RAE) suponga discriminación sexista alguna.

\section{Índice}

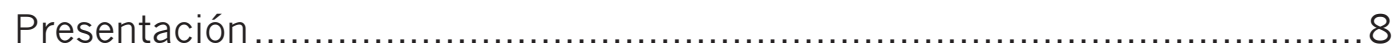

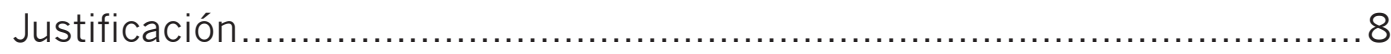

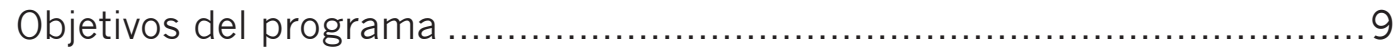

Duración de las actividades .............................................. 9

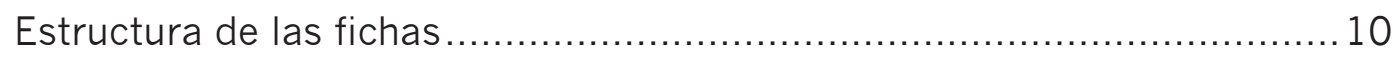

Momento idóneo para utilizar las actividades .................................... 10

Elección de la actividad y lugar para realizarla.................................. 11

Papel de coordinador. Recomendaciones para poner en marcha los descansos activos en tu centro ............................................ 11

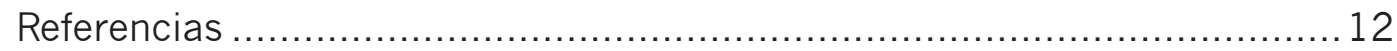

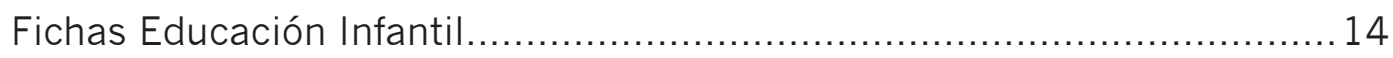

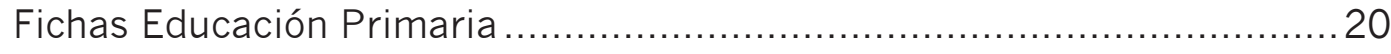

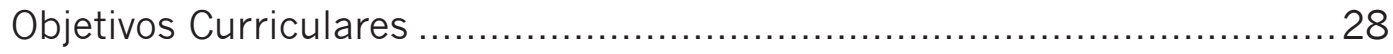




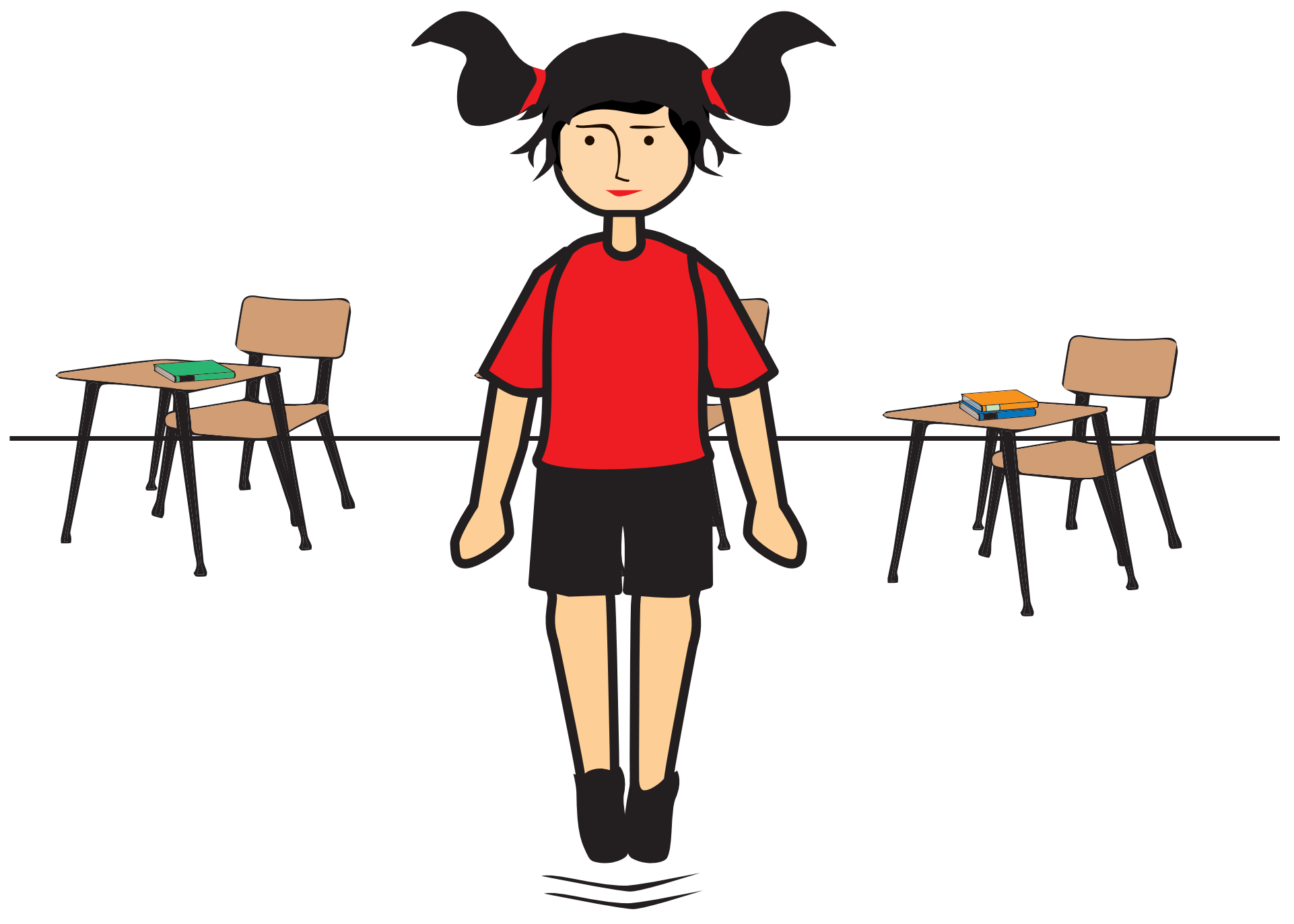





\section{PRESENTACIÓN}

Estos materiales son el resultado de un convenio de colaboración entre la Consejería de Educación, Cultura y Deportes de la JCCM y la UCLM para el desarrollo de programas de fomento de la actividad física y el deporte. Cuyos objetivos fueron:

- Adaptar los materiales originales del programa TAKE10-CHILE! al contexto y currículum educativo de Educación Infantil y Primaria de Castilla-La Mancha.

- Probar la aceptación y viabilidad de la metodología basada en la actividad física (AF) integrada en el currículum por parte de los maestros y centros escolares.

- Elaborar un documento que contenga la metodología y algunos ejemplos de los materiales adaptados para que los centros educativos puedan utilizarlo.

La metodología basada en la AF integrada dentro del currículum se caracteriza por incluir periodos cortos (5.10 minutos) de AF dentro del aula (p.ej., andar, correr o saltar) mientras se aprenden o repasan contenidos académicos de diferentes áreas curriculares (matemáticas, lengua, inglés o ciencias de la naturaleza). Por ejemplo, los niños pueden saltar encima de letras pintadas en el suelo para deletrear una palabra, contar o hacer operaciones matemáticas sencillas a la vez que saltan con una cuerda imaginaria, andar o correr dibujando diferentes trayectorias para representar figuras geométricas (ej.: triángulo, cuadrado), etc. Bajo esta filosofía nace el programa TAKE10! (www.take10.net) que fue desarrollado en 1999 por la Fundación para la Investigación ILSI, con el objetivo de promover la AF estructurada en bloques de 10 minutos dentro del aula, a la vez que se refuerzan los contenidos curriculares. TAKE10! ha sido implementado durante años en más de 40.000 escuelas de Estados Unidos y exportado a otros países como China (Happy10), Brasil (TIRE10!), Chile (TAKE10-CHILE!) y Reino Unido (TAKE10 UK) (1).

Esta metodología ha demostrado un efecto positivo tanto en términos de salud como académicos. Específicamente, ha mostrado ser efectiva para aumentar los niveles de AF moderada-vigorosa, disminuir el tiempo de sedentarismo, controlar el peso corporal, mejorar la forma física, reducir las conductas disruptivas haciendo que los escolares estén más atentos en las tareas, aumentar la diversión en el aula y mejorar la función cognitiva y el rendimiento académico (2-4).

\section{JUSTIFICACIÓN}

Mejorar el rendimiento académico y la salud de los escolares es hoy en día una preocupación emergente. La AF tiene el potencial para abordar ambas cuestiones. Desafortunadamente, actualmente, estamos presenciando una significativa reducción de los niveles de AF en los niños y adolescentes (5). En el sistema educativo 
español, los escolares de Educación Primaria solo reciben 2.3 sesiones de 45 min/ semana o 2 sesiones de 60 min/semana de Educación Física formal. En la etapa de Educación Infantil, en la mayoría de los centros, los niños sólo realizan 1 sesión a la semana de 55 minutos de psicomotricidad, tiempo insuficiente para alcanzar plenamente los objetivos y desarrollar los contenidos relacionados con la motricidad presente en el currículo. Además, se ha descrito que los escolares de esta edad invierten aproximadamente un $80 \%$ de la jornada escolar en actividades sedentarias. Estos datos son preocupantes si se tienen en cuenta las actuales recomendaciones de AF que señalan que los niños de 6.12 años deberían acumular al menos $60 \mathrm{mi}$. nutos diarios de AF de intensidad moderada-vigorosa (6). Y en el caso de niños menores de 5 años, al menos 120 minutos diarios, 60 minutos de AF estructurada y 60 minutos o más de AF no estructurada (actividades recreativas, juegos, o deportes), y reducir los periodos sedentarios prolongados a menos de $1 \mathrm{~h}$ seguida (7).

La presión social por alcanzar los logros académicos ha provocado un estilo de enseñanza centrado en las actividades sedentarias dentro del aula y una reducción de las oportunidades para ser físicamente activo dentro de la escuela. Se ha instaurado la creencia de que el movimiento y la AF no son importantes para alcanzar los logros académicos. Sin embargo, la evidencia científica indica que debería imperar un enfoque totalmente opuesto en los centros escolares, ya que los resultados de las últimas revisiones de la literatura sobre la relación entre la AF y el rendimiento académico han concluido que dar oportunidades a los escolares para que sean físicamente activos dentro y fuera de la jornada escolar puede mejorar el rendimiento escolar, a la vez que mejoran otros aspectos de la salud de los niños $(8,9)$.

\section{OBJETIVOS DEL PROGRAMA}

1. Aumentar los niveles de actividad física diaria y reducir el sedentarismo durante el horario escolar.

2. Favorecer los procesos de aprendizaje a través de la mejora de la concentración, alivio de la tensión y el estrés.

\section{DURACIÓN DE LAS ACTIVIDADES}

Cada actividad tiene una duración entre 5-10 minutos aproximadamente: uno o dos minutos de repaso del contenido a reforzar, tres o cinco minutos de desarrollo y uno o dos minutos de vuelta a la calma. 


\section{ESTRUCTURA DE LAS FICHAS}

Cada actividad incluye, en la parte de la izquierda:

Curso, área de conocimiento, contenido/s, objetivo/s de área (en el caso de Educación Infantil) o generales (en el caso de Educación Primaria), competencias básicas (en el caso de Educación Infantil) o claves (en el caso de Educación Primaria), organización de la clase, así como el espacio para llevarla a cabo.

En la parte de la derecha se detalla:

- Clasificación según grado de intensidad de la AF y según el grado de exigencia cognitiva. Representándose a través de colores (rojo: alta, amarilla: media y verde: baja) y formas (círculo para la intensidad de la AF y un cuadrado para la exigencia cognitiva).

- Contenido a reforzar: breve descripción del contenido que hay que reforzar.

- Desarrollo: actividad propuesta.

En la parte de abajo se describe:

- Vuelta a la calma: actividad para bajar la activación y preparar al alumno para volver a las actividades de clase. Pueden presentarse en forma de actividades que requieren un nivel de AF leve o en forma de preguntas que ayuden a tomar conciencia sobre la importancia de los hábitos saludables.

- Variantes: son adaptaciones para aumentar o disminuir la dificultad sobre la actividad propuesta con el objetivo de adaptarla a diferentes niveles y grupos.

\section{MOMENTO IDÓNEO PARA UTILIZAR LAS ACTIVIDADES}

Cada profesor elegirá el momento de la jornada escolar que mejor se ajuste a él y a los niños. No obstante, se recomienda su utilización cuando el docente observe que sus alumnos lleven mucho tiempo sentados o estén cansados de las tareas rea. lizadas, de tal forma que permita recuperar su atención (recuerda que más de 45.50 minutos sentados reduce los niveles de atención y perjudica la salud).

Para favorecer la coordinación entre los diferentes profesores y motivar a su rea. lización, se puede incluir un horario semanal, que se colocaría en un lugar visible dentro del aula, para indicar en qué momento se ha hecho la actividad. 


\section{ELECCIÓN DE LA ACTIVIDAD Y LUGAR PARA REALIZARLA}

Se realizará la actividad que más se adapte a los contenidos que el profesor quiere trabajar, en función de la clasificación de las sesiones según el área de cono. cimiento. Debe tenerse en cuenta que las actividades propuestas permiten flexibilidad para que el profesor pueda reforzar otro contenido, manteniendo la metodología pero ajustándolo a su programación.

Se recomienda realizar 2 actividades al día, 5 días a la semana con el objetivo de conseguir 100 minutos de AF semanales. Siendo el mínimo recomendado 75 minutos de AF semanales con esta metodología (unas 7 actividades/semana).

Las sesiones no requieren de material específico y se realizarán normalmente en el aula, aunque es posible llevarlas a cabo en el pasillo o incluso en el patio una vez que los alumnos tengan las dinámicas interiorizadas y se minimice el tiempo de organización.

\section{PAPEL DEL COORDINADOR. RECOMENDACIONES PARA PONER EN MARCHA LOS DESCANSOS ACTIVOS EN TU CENTRO}

1.Empieza experimentando en tu propia aula/grupo.

2. Cuando te sientas seguro comparte tu experiencia:

- Invita a tu clase a tus compañeros.

- Pasa a las clases de tus compañeros y realiza un descanso activo.

- Graba vídeos de tus descansos activos y compártelos.

3. Invita a todos los docentes de tu centro, pero sobre todo a los más motivados (se trata de crear poco a poco un grupo motivado que sea capaz de entusiasmar al resto).

4. Proporciona a tus compañeros evidencia científica sólida para convencer con argumentos de peso sobre:

- Niveles actuales de AF de los escolares.

-El aumento significativo de las conductas sedentarias.

- Riesgos asociados al sedentarismo.

- Beneficios para la salud y el rendimiento académico asociados a la AF.

- Beneficios y ventajas de aplicar esta metodología en el aula.

5. Transmite los siguientes mensajes clave. Con este recurso didáctico:

- NO se pierde tiempo, se gana. 
- Mejora la imagen del centro.

- Se trata de cubrir necesidades de los alumnos, por lo que el docente debe analizar estas necesidades y aplicar los recursos y estrategias que mejor va. yan al contexto del grupo clase.

- Mejora el aprendizaje y el bienestar del alumno, por lo que se debería incorporar en la metodología de forma habitual y permanente, no como una experiencia puntual.

6. Promueve conversaciones entre los docentes que lo apliquen para que hablen de sus experiencias y de cómo se sienten sus alumnos.

7. Si el colegio cuenta con los permisos adecuados sube los vídeos al blog del co. legio, esto creará expectación y "buen nombre" para el docente y el centro.

8. Habla con los docentes de Educación Física y acordar varios movimientos básicos que sean idóneos para utilizarse en los descansos activos (que utilicen grandes grupos musculares, que sean funcionales y compensatorios de la postura sentada, impliquen poco desplazamiento tales como las sentadillas, sentarse en el suelo y levantarse, etc.). Podrán trabajar su técnica correcta en una sesión de Educación Física y así se disminuirá el tiempo de explicación en el aula y aumentará su efecto. Recuerda que si las características del aula y del centro te lo permiten puedes proponer también movimientos que requieran desplazamien. tos dentro del aula, o incluso realizar los descansos en los pasillos o patio.

\section{REFERENCIAS}

1. Kibbe DL, Hackett J, Hurley M, McFarland A, Schubert KG, Schultz A. Ten Years of TAKE10!: Integrating physical activity with academic concepts in elementary school classrooms. Prev Med. 2011; 52: S43-S50.

2. Mischo A, Vazou S. Effect of classroom-based physical activity intervention on body mass index and activity levels: A meta- analysis. Med Sci Sports and Exerc. 2014; 46: 231-232.

3. Fedewa AL, Ahn S. The effects of physical activity and physical fitness on children's achievement and cognitive outcomes: a meta-analysis. Res Q Exerc Sport. 2011;82(3):521-35.

4. Webster CA, Russ L, Vazou S, Goh TL, Erwin H. Integrating movement in academic classrooms: understanding, applying and advancing the knowledge base. Obes Rev. 2015;16(8):691-701.

5. Telama, R. Tracking of physical activity from childhood to adulthood: a review. Obes Facts. 2009; 2:187-195. 
6. Recomendaciones mundiales sobre actividad física para la salud. Organización Mundial de la Salud, 2010 [Consultado el 8/04/2016]. Disponible en: http:// whqlibdoc.who.int/publications/2010/9789243599977_spa.pdf.

7. Tremblay MS, Leblanc AG, Carson V, Choquette L, Connor Gorber S, Dillman C, et. al. Canadian Physical Activity Guidelines for the Early Years (aged 0.4 years). Appl Physiol Nutr Metab. 2012;37 (2): 345-69.

8. Diamond AB. The cognitive benefits of exercise in youth. Curr Sports Med Rep. 2015; 14 (4): 320-26.

9. Rasberry CN, Lee SM, Robin L, Laris BA, Russell LA, Coyle KK, et al. The as sociation between school-based physical activity, including physical education, and academic performance: a systematic review of the literatura. Prev Med. 2011;52 Suppl 1:S10-20. 


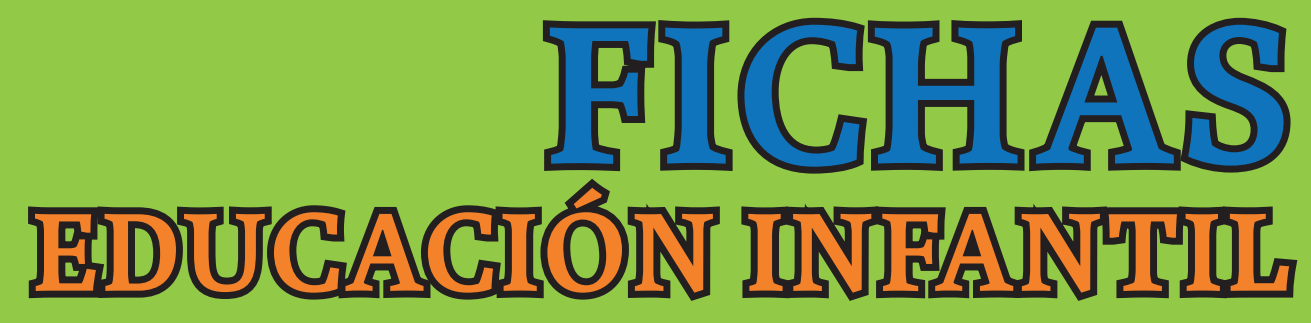









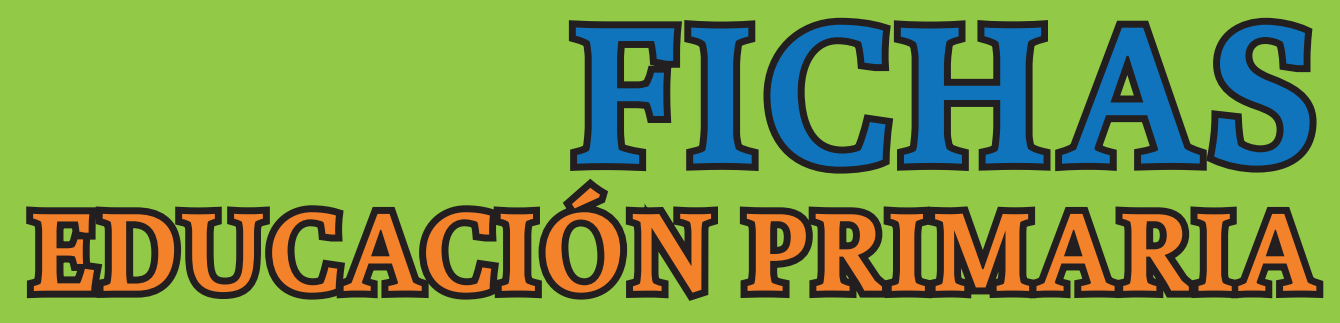






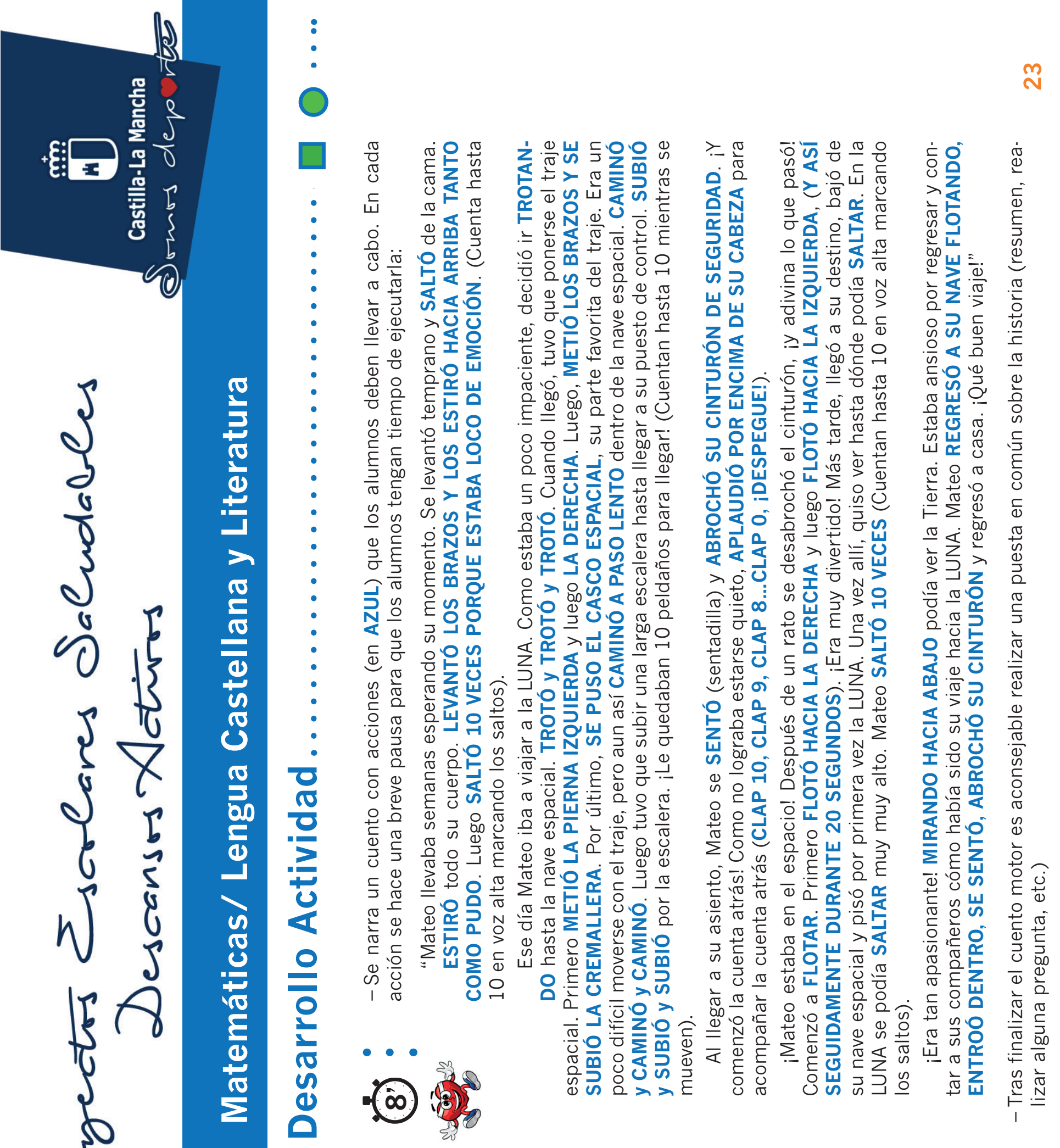

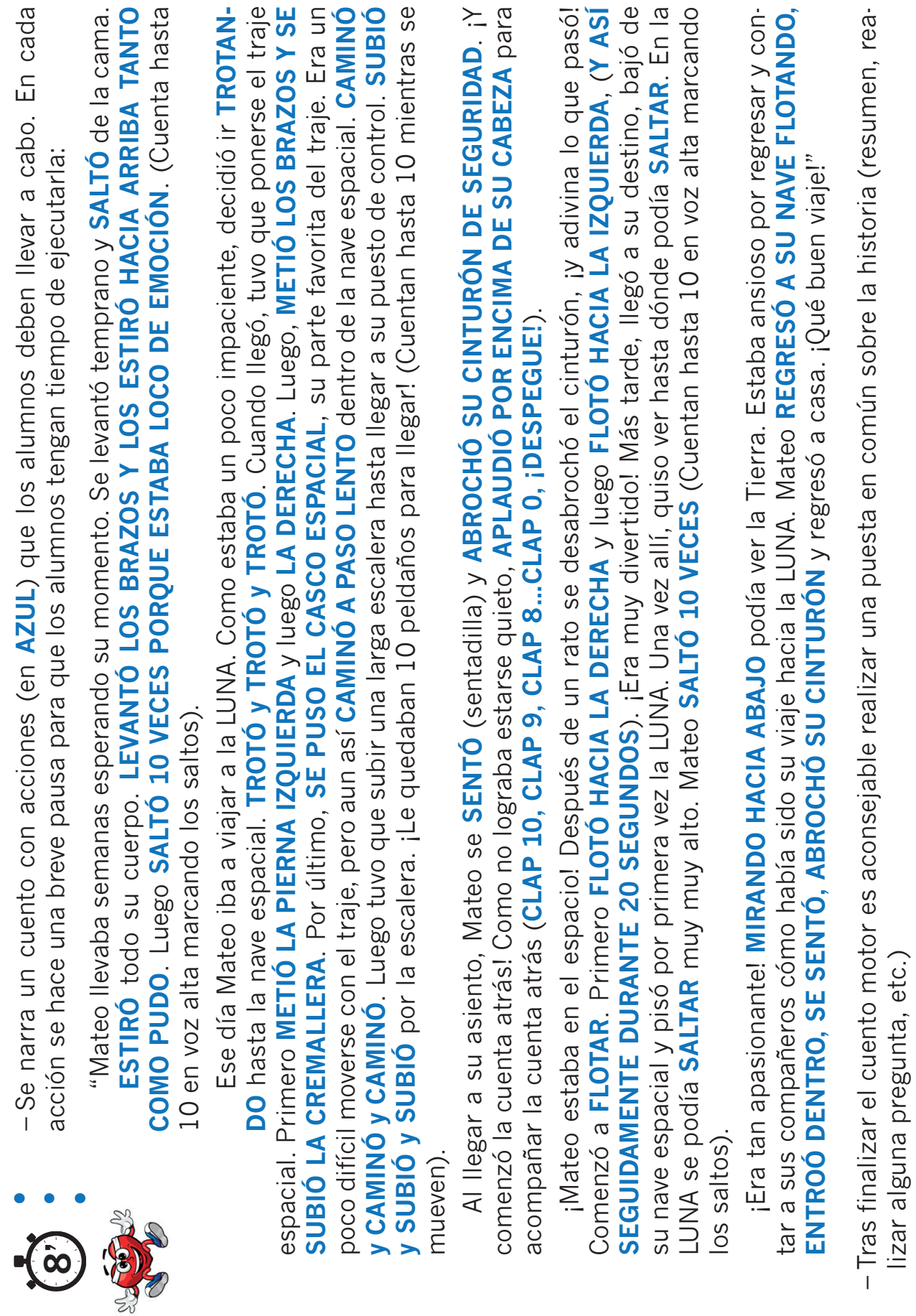
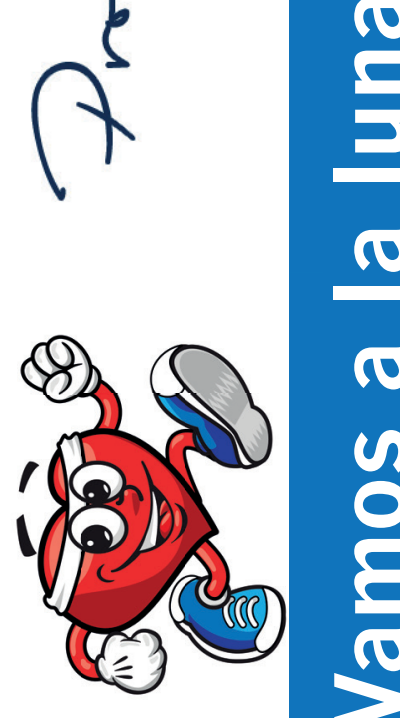

10

$\mathbb{0}$
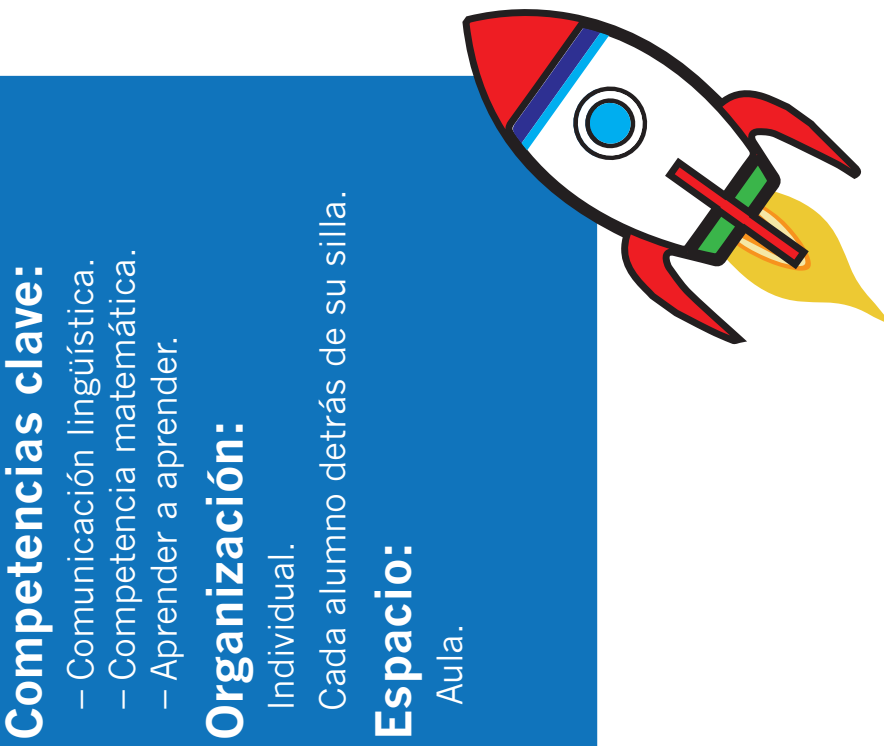


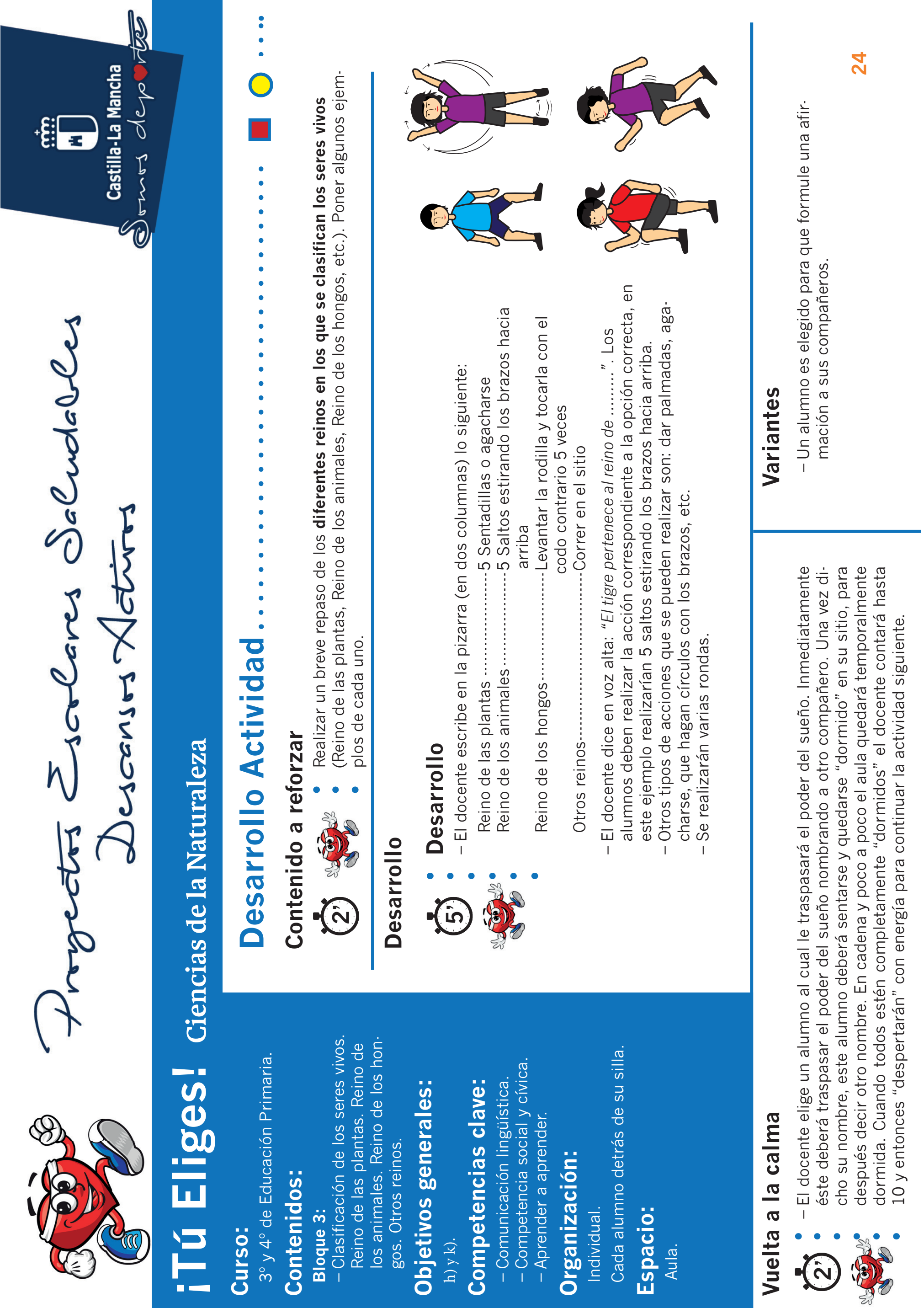






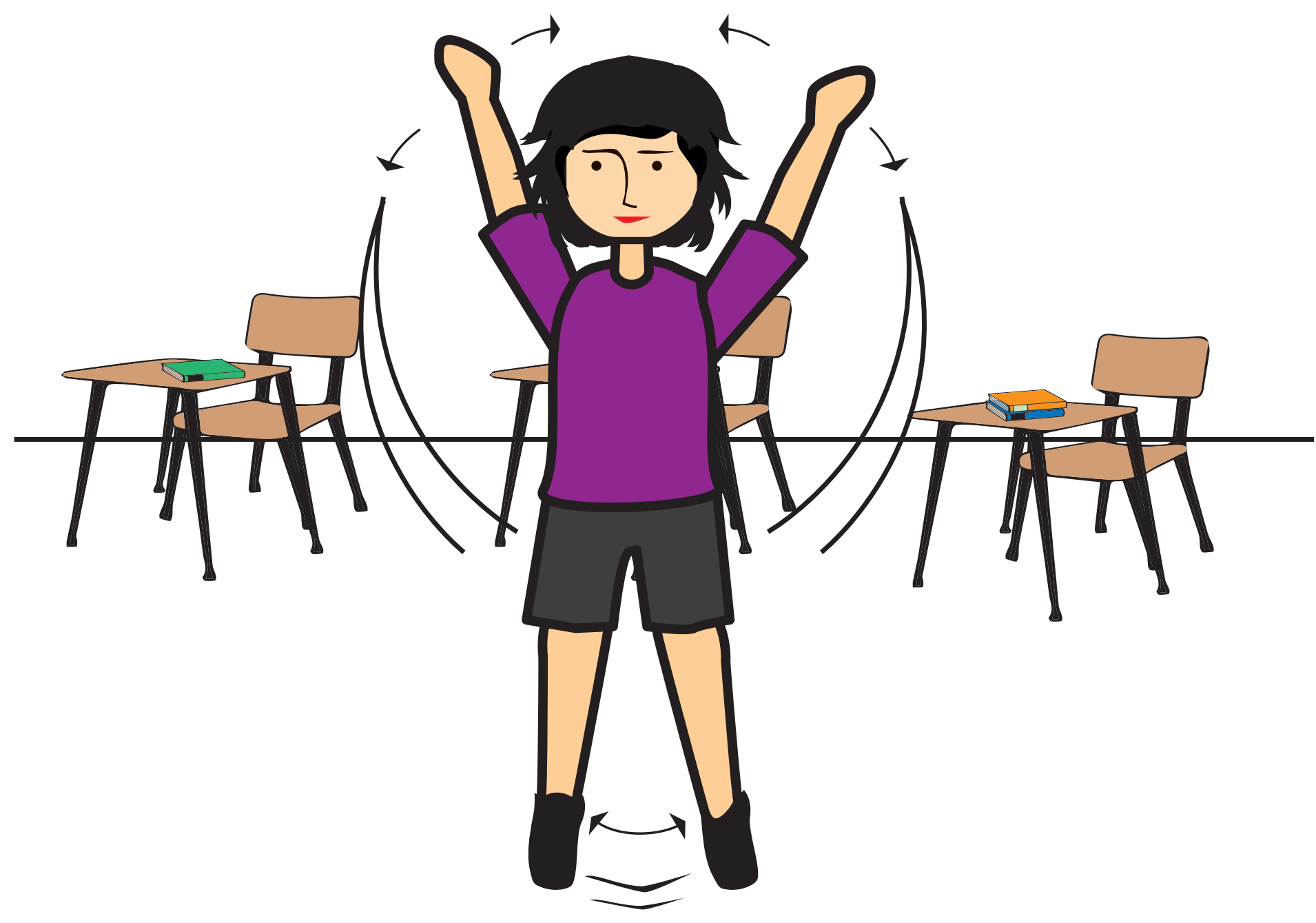




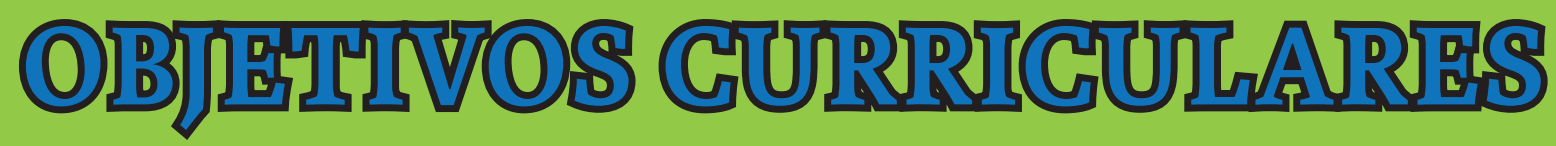




\section{Objetivos de área de Educación Infatil}

\section{Conocimiento de sí mismo y autonomía personal:}

1. Conocer, representar y utilizar el cuerpo, sus elementos, funciones, posibilidades de acción y de expresión de una forma controlada y coordinada.

2. Formarse una imagen ajustada de sí mismo en la interacción con los otros y en el desarrollo de la autonomía personal.

3. Identificar, dominar y comunicar los sentimientos, emociones, necesidades o preferencias propias y conocer, comprender y respetar las de los otros.

4. Realizar con autonomía y seguridad los hábitos personales, las actividades habituales y tener iniciativa para resolver las nuevas tareas y problemas que presenta la vida cotidiana.

5. Desarrollar actitudes de hábitos de respeto, ayuda y colaboración con los demás; de promoción de la salud y de protección del entorno.

\section{Conocimiento e interacción con el entorno:}

1. Observar y explorar con interés el entorno natural para conocer y valorar los componentes básicos e interpretar algunas de sus relaciones y desarrollar actitudes de cuidado, respeto y responsabilidad en su conservación.

2. Iniciarse en las habilidades matemáticas, actuando sobre elementos y colecciones, identificando sus atributos y cualidades y estableciendo relaciones de agrupamientos, clasificación, orden y cuantificación.

3. Relacionarse con los demás, de forma cada vez más equilibrada y satisfactoria, interiorizando progresivamente las pautas de comportamiento social y ajustando su conducta a ellas.

4. Conocer distintos grupos sociales cercanos a su experiencia, algunas de sus características, producciones culturales, valores y formas de vida, generando actitudes de confianza, respeto y aprecio.

\section{Lenguajes: Comunicación y Representación:}

1. Utilizar el lenguaje oral para expresar sentimientos, deseos e ideas, y valorar su uso como herramienta de relación con las demás, de regulación de la convivencia y como instrumento de aprendizaje tanto en lengua propia como extranjera.

2. Comprender las intenciones y mensajes de otros niños y adultos, adoptando una actitud positiva hacia la lengua, tanto propia como extranjera.

3. Iniciarse en los usos sociales de la lectura y la escritura explorando su funcionamiento y valorándolas como instrumento de comunicación, información y disfrute

4. Comprender y disfrutar escuchando, interpretando y leyendo textos literarios mostrando actitudes de valoración, disfrute e interés hacia ellos. 
5. Comprender y representar ideas y sentimientos empleando el lenguaje plástico, corporal y musical mediante el empleo de diversas técnicas y acercarse al conocimiento de obras artísticas expresadas en esos lenguajes.

6. Utilizar el ordenador para acceder al uso del lenguaje multimedia para mejorar o reforzar habilidades y conocimientos.

\section{Objetivos generales de la Educación Primaria}

a) Conocer y apreciar los valores y las normas de convivencia, aprender a obrar de acuerdo con ellas, prepararse para el ejercicio activo de la ciudadanía y res. petar los derechos humanos, así como el pluralismo propio de una sociedad democrática.

b) Desarrollar hábitos de trabajo individual y de equipo, de esfuerzo y de responsabilidad en el estudio, así como actitudes de confianza en sí mismo, siendo crítico, iniciativa personal, curiosidad, interés y creatividad en el aprendizaje, y espíritu emprendedor.

c) Adquirir habilidades para la prevención y para la resolución pacífica de conflictos, que les permitan desenvolverse con autonomía en el ámbito familiar y doméstico, así como en los grupos sociales con los que se relacionan.

d) Conocer, comprender y respetar las diferentes culturas y las diferencias entre las personas, la igualdad de derechos y oportunidades de hombres y mujeres y la no discriminación de personas con discapacidad.

e) Conocer y utilizar de manera apropiada la lengua castellana y desarrollar hábitos de lectura.

f) Adquirir en una lengua extranjera, al menos, la competencia comunicativa básica que les permita expresar y comprender mensajes sencillos y desenvolverse en situaciones cotidianas.

g) Desarrollar las competencias matemáticas básicas e iniciarse en la resolución de problemas que requieran la realización de operaciones elementales de cálculo, conocimientos geométricos y estimaciones, así como ser capaces de aplicarlo a las situaciones de su vida cotidiana.

h) Conocer los aspectos fundamentales de las Ciencias de la Naturaleza, Ias Ciencias Sociales, la Geografía, la Historia y la Cultura.

i) Iniciarse en la utilización, para el aprendizaje, de las tecnologías de la información y la comunicación, desarrollando un espíritu crítico ante los mensajes que reciben y elaboran. 
j) Utilizar diferentes representaciones y expresiones artísticas e iniciarse en la construcción de propuestas visuales y audiovisuales.

k) Valorar la higiene y la salud, aceptar el propio cuerpo y el de los otros, respetar las diferencias y utilizar la educación física y el deporte como medios para fa. vorecer el desarrollo personal y social.

I) Conocer y valorar los animales más próximos al ser humano y adoptar modos de comportamiento que favorezcan su cuidado.

m) Desarrollar sus capacidades afectivas en todos los ámbitos de la personalidad y en sus relaciones con los demás, así como una actitud contraria a la violencia, a los prejuicios de cualquier tipo y a los estereotipos sexistas.

n) Fomentar la educación vial y actitudes de respeto que incidan en la prevención de los accidentes de tráfico. 


\section{Entidades colaboradoras:}

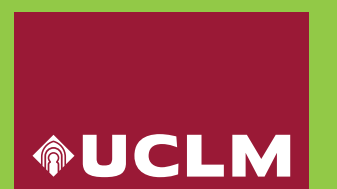

Universidad de

Castilla La Mancha
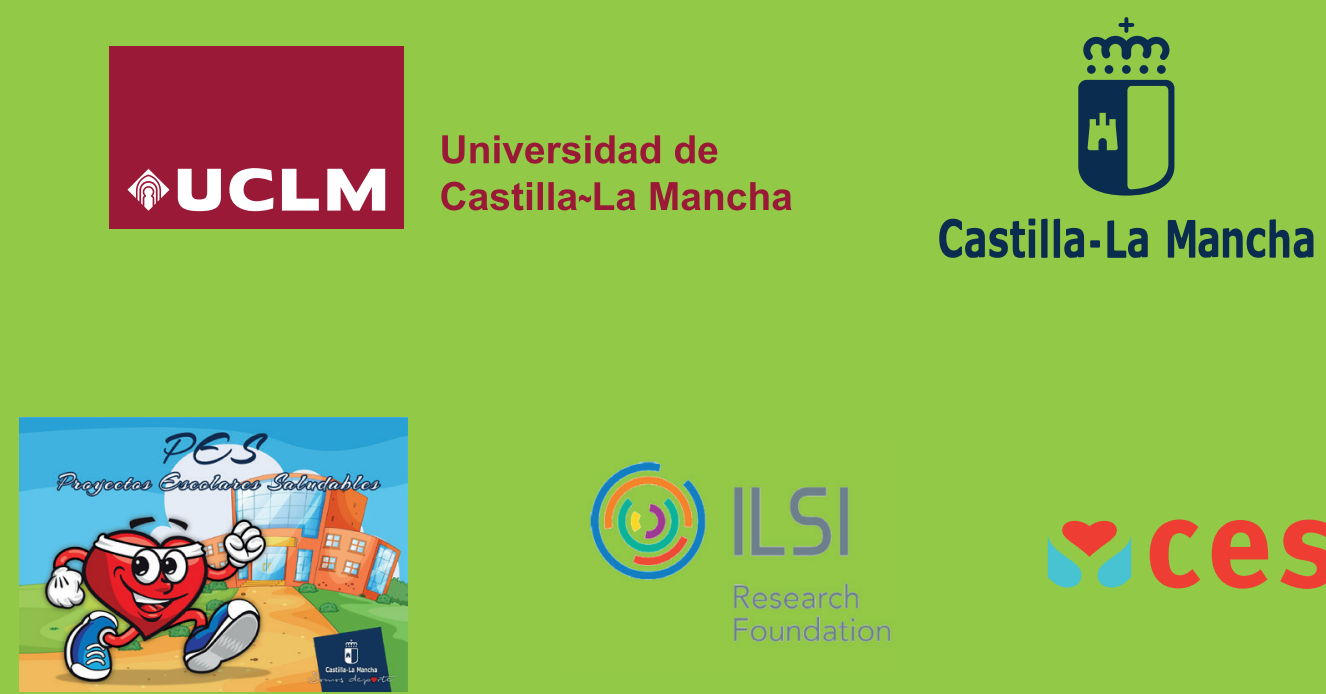

- cesz 